\title{
Die Entmachtung des Teufels. Das Jenaer Ereignis 1715 und die Dämonologie der Aufklärung
}

La destitution du diable. L'événement d'Iéna 1715 : pour une démonologie des Lumières allemandes?

Satan deprived of power. The Jena Affair of 1715 and the Demonology of Enlightenment

\section{Thomas Nicklas}

\section{OpenEdition Journals}

Édition électronique

URL : https://journals.openedition.org/ceg/11853

DOI : $10.4000 /$ ceg. 11853

ISSN : 2605-8359

\section{Éditeur}

Presses Universitaires de Provence

\section{Édition imprimée}

Date de publication : 1 avril 2012

Pagination : 165-178

ISBN : 0751-4239

ISSN : 0751-4239

Référence électronique

Thomas Nicklas, „Die Entmachtung des Teufels. Das Jenaer Ereignis 1715 und die Dämonologie der Aufklärung", Cahiers d'Études Germaniques [Online], 62 | 2012, Online erschienen am: 12 Oktober 2020, abgerufen am 07 Juni 2021. URL: http://journals.openedition.org/ceg/11853 ; DOI: https://doi.org/ 10.4000/ceg. 11853 


\title{
Die Entmachtung des Teufels. Das Jenaer Ereignis 1715 und die Dämonologie der Aufklärung
}

\author{
Thomas NICKLAS \\ Université de Reims - Champagne Ardenne
}

In Theodor Storms 1878 erschienener Novelle Renate tritt in der Gestalt des Pastors Petrus Goldschmidt (1662-1713) ein leidenschaftlicher Verteidiger des Hexen- und Teufelsglaubens an der Wende vom 17. zum 18. Jahrhundert auf, dessen „gewaltige und robuste Gottesgelahrtheit“ im Stormschen Novellentext ein ironisch gemeintes Lob erfährt. ${ }^{1}$ Tatsächlich hatte der seit $1691 \mathrm{im}$ schleswigschen Sterup wirkende Pastor Goldschmidt mit großer Entschiedenheit gegen Zweifel an der Hexerei und der Macht des Teufels angekämpft. ${ }^{2}$ So polemisierte er nicht nur heftig gegen den wichtigsten Vertreter cartesianischer Theologie in den Niederlanden, Balthasar Bekker (1634-1698), dessen geisterkritisches Werk Bezauberte Welt 1693 in einer deutschen Übersetzung erschienen war, ${ }^{3}$ auch Christian Thomasius wurde von ihm angegangen, weil der Hallenser Professor in der 1701 veröffentlichten Schrift De crimine magiae die Möglichkeit des Teufelsbündnisses in Abrede gestellt hatte. Dagegen wollte Goldschmidt an der "schändliche[n] und ewig verderbende[n] Bündnis-Stifftung zwischen Teuffeln und Menschen" festgehalten wissen. ${ }^{4}$ Mochte der Pastor aus Sterup mit seinen leidenschaftlichen Ausbrüchen gegen Autoritäten auch seinem Anliegen selbst schaden, so sah er doch in einem Punkt ganz richtig. In der langen Geschichte des "Abschiedes vom Teufel" war um 1700 definitiv ein

1 Theodor Storm, Sämtliche Werke in vier Bänden, Bd. 3, Berlin/ Weimar, AufbauVerlag, ${ }^{4} 1978$, S. 73-137 (hier 109).

2 Vgl. dazu: Jan Ulbe TERPSTRA, "Petrus Goldschmidt aus Husum. Ein nordfriesischer Gegner Balthasar Bekkers und Thomasius", in: Euphorion 59 (1965), S. 361-383.

3 Zu Bekkers "cartesianischer" Theologie: Andrew FIX, Fallen Angels: Balthasar Bekker, Spirit Belief, and Confessionalism in the Seventeenth-Century Dutch Republic, Dordrecht, Kluwer Academic Press, 1999; Wiep van Bunge, "Balthasar Bekker's Cartesian Hermeneutics and the Challenge of Spinozism", in: The British Journal of the History of Philosophy 1 (1993), S. 55-80.

4 Gustav RoskofF, Geschichte des Teufels, Bd. 2, Leipzig, Brockhaus, 1869, S. 483. 
Wendepunkt erreicht. ${ }^{5}$ Nach der unter anderem von Bekker vertretenen "cartesianischen" Auffassung hatten der Teufel und andere geistige Wesen keinerlei Wirkungsmöglichkeiten in der materiellen Welt. Geister waren demnach unvermögend, auf Materie einzuwirken. Damit drohten weitreichende Konsequenzen. Der Leibhaftige wurde zwar nicht beseitigt, aber entmachtet.

Der nicht nur von Goldschmidt artikulierte Widerspruch gegen diesen offenkundigen Machtverlust Satans bestimmte die Strukturen der Teufelsdebatten im Heiligen Römischen Reich am Anfang des 18. Jahrhunderts. Es wäre aber irrig, wollte man nur Theologen und Prediger als Verteidiger der Vorstellung eines mächtigen Teufels identifizieren. Vielmehr wird bei genauerem Hinsehen auch ein Kompetenzstreit zwischen Theologen, Medizinern und Juristen erkennbar, die sich um die Deutungshoheit über Macht und Ohnmacht des Leibhaftigen stritten. So wird das bemerkenswerte mediale Echo verständlich, das ein Unglücksfall hervorrief, der sich zur Weihnachtszeit des Jahres 1715 am Rand der thüringischen Universitätsstadt Jena ereignete. Diese große Aufmerksamkeit für einen bizarren Vorgang hat auch mit der Ausrichtung der Kommunikationsstrukturen des vormodernen Reiches auf das thüringisch-sächsische Zentrum zu tun, in dem das Geschehen stattfand. Das als "Jenaische Christnachtstragödie" viel beachtete und viel beredete Vorkommnis löste eine Kettenreaktion von Stellungnahmen aus, die Rückschlüsse auf Motivationen, Mechanismen und Aporien des Teufels-Diskurses im protestantischen Mitteldeutschland nach 1700 erlauben. ${ }^{6}$ Dabei ist zu bedenken, dass es gerade in Jena Widerstände gegen die Entmachtung des Teufels gab. Einer der bedeutendsten Repräsentanten der Jenenser lutherischen Theologie des 17. Jahrhunderts, Johannes Musaeus (1613-81), hatte sich gegen die Überhandnahme cartesianischer Auffassungen gestemmt und deren Eindringen an der thüringischen Landesuniversität auf Jahrzehnte verhindert. ${ }^{7}$ Es ist daher nicht allzu verwunderlich, wenn eine Diskussion um Macht und Unmacht des Teufels ihren Ausgang von einem Ereignis nahm, das sich am 24./25. Dezember 1715 vor den Toren der Stadt Jena zugetragen hatte.

Ein Medizinstudent namens Johann Gottfried Weber hatte sich in der kalten Winternacht zusammen mit dem Schäfer Johann Friedrich Geßner und dem Bauern Hanns Zenner in das im Jenenser Weinberg gelegene Häuschen des Schneiders Georg Heuchler zurückgezogen, um dort die Geister zu beschwören, die sie zu den im Berg verborgenen Schätzen führen sollten. ${ }^{8}$

5 Der Begriff bei: Marc VenARD, Die Geschichte des Christentums: Das Zeitalter der Vernunft (1620/30-1750), Freiburg, Herder, 1998, S. 1143.

$6 \mathrm{Zu}$ Analyseformen von Diskursen in der Soziologie sei hier nur äußerst knapp verwiesen auf: Reiner KELLER, Wissenssoziologische Diskursanalyse. Grundlegung eines Forschungsprogramms, Wiesbaden, VS, ${ }^{3} 2011$, bes. S. $179 \mathrm{ff}$.

7 Handbuch der Dogmen- und Theologiegeschichte, Bd. 3: Die Lehrentwicklung im Rahmen der Ökumenizität, Göttingen, Vandenhoeck \& Ruprecht, 1984, S. 95.

$8 \mathrm{Zu}$ dem Ereignis selbst und seiner Deutung im Zusammenhang mit ähnlichen Vorgängen im sächsischen Erzgebirge: Gabor RYCHLAK, Hexenfieber im Erzgebirge. Die Annaberger 
Die Beschwörung nahm allerdings einen tragischen Verlauf. Am nächsten Tag fand man den Studenten ohnmächtig in der Hütte auf, Geßner und Zenner waren beide tot. Während der besinnungslose Student Weber in die Stadt gebracht wurde, ordnete der Magistrat drei Wächter ab, die darauf Acht geben sollten, dass die Angehörigen der beiden Toten es nicht unternahmen, noch vor der amtlichen Untersuchung des Falles die Leichen fortzuschaffen. Wegen der starken Kälte zogen sich die Wächter jedoch in die Hütte zurück, in der noch ein Feuer in einem Kohlenbecken glomm. Dort verloren auch sie das Bewusstsein. Zwei von ihnen kamen wieder zu sich, während der dritte nur noch tot aus der Hütte geschafft werden konnte. Den Zeitgenossen erschien bei diesen unerklärlichen Vorgängen ein Einwirken des Teufels überaus plausibel, während im gelehrten Diskurs zumeist eine Kohlenmonoxid-Vergiftung der Opfer als wahrscheinlich angenommen wurde, da die Geisterbeschwörer in der engen und unbelüfteten Hütte ein Holzkohlenfeuer entzündet hatten. Darüber hinaus wurde auch vermutet, dass Weber und seine Konsorten bei ihrem Geschäft giftige Räucherstoffe in dem Kohlenbecken verbrannt hatten. Dies kann als sehr wahrscheinlich angesehen werden, klagten doch sowohl der überlebende Student als auch die beiden Wächter nach ihrer Bergung über Kopfschmerzen und starke Übelkeit.

\section{Auftakt zur Debatte: Die Jenaer Christnachtstragödie als vermutliches Teufelswerk}

Am 6. Januar 1716 erschienen in Leipzig die ersten gedruckten Berichte zu den Vorkommnissen, die sich am Weihnachtsabend in dem Häuschen des Schneiders Heuchler im Jenaer Weinberg zugetragen hatten. ${ }^{9}$ Dabei finden sich bereits alle Elemente vereint, die den späteren Verlauf der Debatte bestimmen sollten. Der anonyme Herausgeber urteilt in seiner Vorrede, dass die Jenenser Begebenheit die Wirkmächtigkeit der Geister erweise:

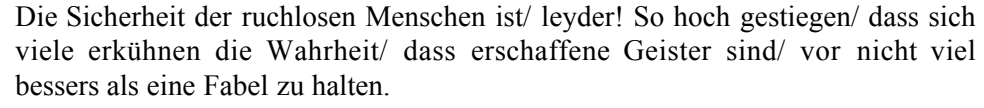

Die Sicherheit der ruchlosen Menschen ist/ leyder! So hoch gestiegen/ dass sich viele erkühnen die Wahrheit/ dass erschaffene Geister sind/ vor nicht viel bessers als eine Fabel zu halten.

Ein abgedruckter erster Bericht aus Jena vom 26. Dezember 1715 hält die Gerüchte unmittelbar nach dem Bekanntwerden des Ereignisses fest. Demnach soll ein Student namens Weber von Reichenbach den Teufel zitiert haben, welcher ihn "bei Arm und Bein genommen und auf den Boden geschmissen". Ein nicht genannter Berichterstatter, ebenfalls aus Jena, fügt

Krankheit 1712-1720, Diss. Mainz 2009, S. 205-211 [http://ubm.opus.hbz-nrw.de/volltexte/ 2009/2159/pdf].

9 Vorläuffige Erste Nachricht Von der entsetzlichen Begebenheit, Die sich den 24. Dec. 1715, Als am Heil. Christ-Abende, in Jena Von Anfang biß den 4. Jan. 1716 Mit Etlichen verwogenen Personen, So den Satan beschworen und citiret, zugetragen, Leipzig, Boutique zum Comtoir-Calender, o.J. [1716]. 
unter dem Datum des 30. Dezember 1715 jene fabelhaften Ausschmückungen hinzu, die sich bereits im öffentlichen Gespräch an das erzählte Faktum angelagert hatten. Demnach sei Weber ein Geist "in Gestalt eines Knaben erschienen und hat von ihm begehrt, sich zu unterschreiben. Als er es nicht getan, bei linker Hand und Fuß angefasset und in die Höhe gehalten, daß ihm der Ellbogen ausgedreht ist." Die Tragödie wird somit als Strafe wegen des verweigerten Teufels-Paktes gedeutet. Nicht recht erklärlich schien es den Berichterstattern jedoch, dass die beiden Mitgesellen Webers, Geßner und Zenner, den Spuk mit dem Leben bezahlt hatten. Ihre Leichen lägen, so hieß es, unterdessen im Jenenser Pestilenz-Haus und die Mäuse fräßen daran.

Ein anderer Verfasser geht unter dem Datum des 31. Dezember 1715 ausführlich auf das inzwischen bekannt gewordene Schicksal der drei Wächter ein, von denen einer aus der Bewusstlosigkeit nicht mehr erwacht war. Allerdings nimmt dieser Bericht kein teuflisches Einwirken an, vielmehr wird vermutet, dass die Schatzsucher beim Räuchern im Weinbergshäuschen giftige Materien verwendet hätten und dass die drei Toten daran erstickt waren. Der letzte Text in der Berichtsammlung, aus Jena am 3. Januar 1716 abgeschickt, geht auf den Inhalt der Predigten ein, mit denen die Jenenser Geistlichkeit inzwischen zu dem Ereignis Stellung genommen hatte. Für die lutherischen Theologen der thüringischen Universitätsstadt stand demnach außer Frage, dass es sich um Teufelswerk gehandelt haben musste. Der Teufel habe dieses angerichtet, vermittels "göttlicher Permission ob der Schändung der Christnacht" durch die Geisterbeschwörer. Somit beinhaltete um die Jahreswende 1715/16 die sich in konzentrischen Kreisen um Jena verbreitende Diskussion über das Ereignis bereits die wesentlichen Elemente des späteren gelehrten Diskurses. Diese reichten von der "übernatürlichen" Annahme einer Gewalttat des Teufels, der die vermessenen Schatzsucher für die Schändung der Christnacht bestrafen sollte, bis zur "natürlichen" Hypothese der Vergiftung durch schlecht dosierte Räuchermittel, die den am eigenen Ungeschick gescheiterten Geisterbeschwörern zum Verhängnis geworden waren.

Da das Publikum weitere Aufklärung forderte, wurde in den ersten Tagen des Jahres 1716 auch in Jena die Druckerpresse angeworfen. Die von dort verbreitete "Relation" konnte aber zu den Vorgängen im Weinbergshäuschen nicht mehr viel Neues beitragen und bot daher vor allem Stoff über die Vorgeschichte der tragisch endenden Beschwörung, aus der inzwischen weitere Details bekannt geworden waren. ${ }^{10}$ Demnach erschien nicht der Student Weber, sondern der Schäfer Geßner als die treibende Kraft bei der

10 Wahrhafftige Relation dessen, was in der Heil. Christ-Nacht [...] 1715 allhier bey der Stadt Jena in einem dem Galgen nah-gelegenen Weinberge mit einer schändlichen Conjuration und Beschwerung deß Satans [...] sich zugetragen hat, Jena, Werther, 1716. Diese Schrift wurde auch unter anderem Titel und mit dem Datum 12. Januar 1716 in Leipzig nachgedruckt: Warhafftige und umständliche fernere Nachricht von der Beschwerung des Satans/ welche ein Studiosus nebst zwey Bauern in Jena vorgenommen, Leipzig, Boutique zum Contoir-Calender, 1716. 
Geisterbeschwörung. Dieser habe nämlich von einem Jenenser Bürger in Erfahrung gebracht, dass "im Weinberg beim Galgen eine weiße Frau öfter erscheine" und daraus geschlossen, dass "ein Schatz aus Kriegszeiten da vergraben" sein müsse. Geßner habe daraufhin den Medizinstudenten angesprochen, da er "sich jemand gewünscht, der die Bücher von der GeistKunst" lesen könne, um eine Beschwörung zu machen. War damit der eigentlich Schuldige benannt, so hatte sich in Jena nach der Tragödie der Christnacht nicht mehr viel ereignet. Freilich hatte noch am 26. Dezember der Medizinprofessor und Stadtarzt Johann Adolph Wedel (1675-1721) die Leichen Geßners und Zenners untersucht und dabei keine Hinweise auf eine äußere Gewalteinwirkung festgestellt. Die zuständige Regierung in Eisenach ordnete an, dass die beiden Körper der Geisterbeschwörer zur Strafe unter dem Galgen verscharrt werden sollten, während der in Ausübung seines Amtes ums Leben gekommene Wächter ehrlich auf dem Kirchhof zu bestatten war. Die beiden überlebenden Wächter klagten, wie hinzugefügt wurde, weiterhin über "Schwachheit des Hauptes". Die Eisenacher Regierung hatte unterdessen zwei Beamte zur Untersuchung des Falles nach Jena gesandt. Über diese amtlichen Nachforschungen drang wenig nach außen, so dass sich spätere Veröffentlichungen zu der Sache auf die Wiederholung bekannter Einzelheiten beschränkten oder Gerüchte kolportierten. Allenfalls erfährt man noch, dass am 11. Januar 1716 die nur spärlich mit Lumpen bedeckten Leichen der Geisterbeschwörer Zenner und Geßner durch die Stadt geschleift und unter dem Galgen in einem besonders tiefen Loch vergraben worden waren. ${ }^{11}$ Damit schien die Gerechtigkeit zumindest nach außen hin wieder hergestellt.

Angesichts der reichsweiten Beachtung, die der Fall auf sich zog, schien es auch Herzog Johann Wilhelm von Sachsen-Eisenach angezeigt, mit einer Veröffentlichung das Informationsbedürfnis des Publikums zu befriedigen. Dem Landesherrn der Stadt Jena war dabei der Nachweis wichtig, dass die amtlichen Untersuchungen korrekt geführt worden waren und die Eisenacher Behörden sich in der Causa nichts vorzuwerfen hatten. ${ }^{12}$ Der amtliche Bericht schöpfte aus den Verhörprotokollen des Studiosus Weber und des Jenaer

11 Fernerer Verfolg Derer Nachrichten Von der, zu Ausgang des 1715ten Jahrs, in der Heil. Christ-Nacht vorgenommenen schändlichen Conjuration, Oder Beschwerung des Satans und darauf erfolgten sehr remarquablen Casu tragico zu Jena, o.O. 1716; die damit an das Publikum gebrachten Informationen wurde in einer ganzen Reihe ähnlicher Schriften weiter verbreitet, siehe u.a.: Die sonderbaren Gerichte Gottes, welche sich Anno MDCCXV. in der Heiligen Christ-Nacht [...] in einem Weinberge der Stadt Jena [...] ereignet, o.O. o.J. [1716]; Erschreck- und entsetzliche Mord-Geschichte, welche der leidige Satan in [...] Ermordung etlicher Personen in der Christ-Nacht [...] zu Jena verübet hat, o.O. o.J. (1716); Wahrhafftige Relation (Anmerkung 10).

12 Wahre Eröffnung der Jenaischen Christnachts-Tragödie: Oder Gründlicher und Actenmäßiger Bericht von der sonderbahren und höchst betrübten Begebenheit, welche in einem der Stadt Jena nahegelegenen Weinbergs-Häusgen mit drey Personen, So die Geister, zur Zeigung eines eingebildeten Schatzes, citiret und beschwohren, im Jahre 1715 in der Christnacht und die folgende Nacht hierauf mit dreyen dahin gesendeten Wächtern sich zugetragen, Jena, Pohl, 1716 [insgesamt drei Auflagen]. 
Bürgers Heuchler, der den Geisterbeschwörern sein Häuschen im Weinberg zur Verfügung gestellt hatte. Beide hatten es offensichtlich gut verstanden, die Schuld weitgehend auf den toten Geßner abzuwälzen. Dieser habe seit längerer Zeit schon verschiedene Personen angesprochen, da er um einen Schatz wüsste und zu dessen Hebung nur noch "die Springwurzel und Dr. Fausts Buch vom Höllen-Zwang" benötigte. Der Jenenser Stadtphysikus Johann Adolph Wedel, der die Leichen untersucht hatte, wurde gegen jede Kritik in Schutz genommen. Ausdrücklich heißt es in der amtlichen Veröffentlichung, dass er auch die Kohlen aus dem im Weinbergshäuschen aufgestellten Becken untersucht und dabei nichts Auffälliges bemerkt habe. Die zentrale Frage, ob bei dem Unglück der Satan Hand angelegt habe oder ob es natürlichen Umständen zuzuschreiben sei, entscheidet die "Wahre Eröffnung" bewusst nicht. Die juristisch geschulten Verfasser fordern jedoch ausdrücklich ein Verbot der Schatzgräberei im Heiligen Römischen Reich, da es sich dabei um die eigentliche Wurzel des Übels handle.

\section{Die Deutungshoheit der Mediziner in Teufelsdingen: Halle gegen Jena}

Damit waren alle Elemente in die Debatte eingeführt und der Sachverhalt hinlänglich bekannt geworden, so dass ein gelehrter Schlagabtausch in Gang kommen konnte, der sich innerhalb des mitteldeutschen Städte- und Hochschuldreiecks Jena/Halle/Leipzig abspielte, wobei die Mediziner noch vor Theologen und Juristen die Deutungshoheit in Teufelsfragen für sich beanspruchten. In Halle lehrte der höchst angesehene und zeitweilig als Leibarzt des preußischen Königs Friedrich I. in Berlin tätige Mediziner Friedrich Hoffmann (1660-1742), der eine große Anzahl von Schülern um sich gesammelt hatte. ${ }^{13}$ Der als gelehrte Autorität anerkannte Hoffmann wurde um seine Meinung zu den Aufsehen erregenden Jenenser Vorfällen angegangen. Seine schriftliche Stellungnahme wurde daraufhin sogleich im Druck veröffentlicht, allerdings ohne Verfassernamen. ${ }^{14}$ Für den Hallenser Universitätskollegen von Christian Thomasius war es klar, dass die "Tragödie" nur natürliche Ursachen haben konnte. Alle anders lautenden Vermutungen waren demnach widersinnig:

13 Neue Deutsche Biographie 9 (1972), S. 416-418; vgl. auch: Gotthold MAMLOCK, "König Friedrich Wilhelm I. Briefe an den Hallenser Kliniker Friedrich Hoffmann", in: Deutsche Medizinische Wochenschrift 37 (1911), S. 2242-2244.

14 Eines berühmten Medici Gründliches Bedencken und physicalische Anmerckungen Von dem tödlichen Dampff der Holtz-Kohlen: Auf Veranlassung der in Jena beym Ausgang des 1715. Jahrs vorgefallenen traurigen Begebenheit aufgesetzet, Halle, Renger, 1716. Eine allerdings nicht zuverlässige Edition der Schrift in: Albert NEUBURGER (Bearb.), Friedrich Hoffmann über das Kohlenoxydgas: Und die Gegenschrift von Andreas Erdmann: "Wie nicht Kohlenmonoxydgas, sondern der Teufel den Tod etlicher Menschen herbeigeführt", Leipzig, Voigtländer, 1912 (=Voigtländers Quellenbücher, 14). 


\begin{abstract}
Man würde sich aber sehr übereilen/ wenn man glauben wollte/ als wenn der böse Feind/ auf Gottes Zulassen/ durch eine unbekannte und verborgene Art diese Leute getödtet hätte.
\end{abstract}

Vielmehr hätten sie sich durch ihre Geldgier und den Aberglauben an die Macht des Teufels, der Reichtum verschaffen könne, selbst zugrunde gerichtet. Einen Sinn konnte das Ereignis für Hoffmann nur dann haben, wenn es als Warnung aufgefasst wurde, beim Entzünden von Holzkohle große Versicht walten zu lassen:

Man hüte sich für den Dampf von feuchten nicht genug ausgebrannten und erst anglimmenden Kohlen/ als für eine Sache/ die leichter und unvermerckter/ als das stärckste Gifft tödten kann.

Er verweist darauf, dass bereits die Antike um die Gefährlichkeit des Kohlenfeuers wusste und bringt zahlreiche Beispiele, um seine These zu stützen. Die Teufels-Hypothese schien Hoffmann in diesem Zusammenhang so wenig plausibel, dass er in seiner Stellungnahme davon absah, sich mit ihr zu befassen.

Dies reizte diejenigen zum Widerspruch, die in den Vorfällen einen Beweis für die Macht des Teufels sehen wollten. Der in Jena praktizierende Mediziner Erdmann Friedrich Andreae sah sich zu einer Gegenpublikation veranlasst. $^{15}$ In dem Verfasser der angegriffenen Schrift vermutete er nicht den berühmten Medizinprofessor Hoffmann, sondern einen unbelehrbaren Cartesianer vom Schlage Bekkers, gegen den sich leicht vorgehen ließ, da er sich als Mediziner zu erkennen gab. Der Jenenser Andreae vertrat folglich die Gegenthese, dass Geister sehr wohl auf die Materie einwirkten. Der Teufel konnte sich auch der Materie bedienen, um sein Werk zu verrichten: "Denn er ist ja ein mächtiger Geist/ der auff tausenderley Art und Weise sich der natürlichen Dinge gebrauchen/ und darmit den Menschen Schaden zufügen kann.” Die Hallenser Schrift sei überaus schädlich, lehre sie den Leser doch den Glauben an einen "ohnmächtigen Teufel" und gehe nur von natürlichen Ursachen aus. Da es sich um eine für die öffentliche Moral gefährliche Lehre handle, komme man auch nicht umhin, die Redlichkeit des Verfassers selbst in Zweifel zu ziehen. Dieses Argument ad personam und das weitschweifige Plädoyer aus Jena für die Macht des Teufels wurde in Halle übel aufgenommen. Friedrich Hoffmann sah sich persönlich angegriffen, obwohl seine Schrift anonym erschienen war. Einer seiner zahlreichen Schüler, der später von Hoffmann in jeder Weise protegierte Johann Heinrich Schulze (1687-1744), schritt daher, von seinem Lehrer ausdrücklich dazu ermutigt, zu

15 Erdmann Friedrich ANDREAE, Gründlicher Gegensatz auf das ohnlängst zu Halle im Magdeburgischen ausgegebene gründliche Bedencken und physicalische Anmerckungen eines berühmten Medici von dem tödtlichen Dampfe der Holtz-Kohlen, Jena, Werther, 1716. Zu Andreae: Ernst GIESE/ Benno von HAGEN, Geschichte der Medizinischen Fakultät der Friedrich-Schiller-Universität Jena, Jena, Fischer, 1958, S. 169-171. 
einer Gegenveröffentlichung. ${ }^{16}$ In seiner Vorrede weist Schulze vorsorglich darauf hin, dass Hoffmann von verschiedenen Seiten Unterstützung erfahren habe, sogar von den Hallenser Theologen, die aber in der Sache nur als Hilfstruppe der debattierenden Mediziner erscheinen. In der hauptsächlichen Streitfrage um die teuflische Macht oder Ohnmacht bezieht der angehende Arzt einen klaren Standpunkt gegen den Jenaer Angreifer:

Nun aber kann alles/ was bey dem Jenischen Casu ungewöhnlich scheinet/ von causis manifestis \& naturalibus klar und deutlich hergeleitet werden: folglich thäte man übel/ woferne man auf ausserordentliche/ geistliche und verborgene verfiele/ oder dieselbe mit Haaren herbey zöge.

Der Cartesianismus-Vorwurf Andreaes richte sich von selbst, da daran nichts Verwerfliches sei! Im Gegenteil sollten alle Mediziner auch im Sinne Descartes' um eine gründliche Einsicht in die Natur bemüht sein, "damit sie bey vorfallenden ungemeinen Begebenheiten nicht so gleich stutzen und auf allerhand unnöthige Grillen verfielen/ oder gar die Medicinische Station verlassen/ und in der duncklen pneumatica ein asylum ignorantiae suchen müsten.” Es gebe keinen einzigen klaren Beweis dafür, dass der Teufel die Macht habe, einen Menschen zu töten. Daher könne von dieser Hypothese vernünftiger Weise nicht ausgegangen werden. Selbst wenn es einmal gelänge, solche Beweise beizubringen, "so blieben doch bey gegenwärtigem Jenaischen casu unendlich viele Schwürigkeiten übrig/ biß man alle natürliche Ursachen von dem erfolgten effectu removirete/ und die Sache dem bösen Feinde in solidum vindicirte.” Es sei folglich barer Unsinn, mit der These von der Macht des Teufels zu operieren.

Freilich geht der Kandidat der Medizin Schulze aus Halle in seiner Erwiderung noch einen Schritt weiter. So behauptet er gegen Andreae, dass es sogar ein der Religion dienliches Werk sei, die Macht des Teufels zu brechen, die mit dem Glauben an sie hinweg fiele. Diese Macht rühre nur von jenen her, die an ihn glaubten. Aus Gottesfurcht werde so Teufelsfurcht:

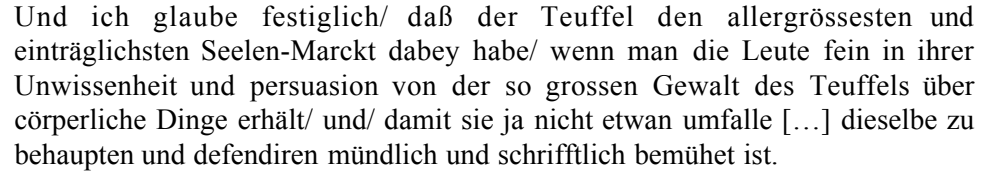
einträglichsten Seelen-Marckt dabey habe/ wenn man die Leute fein in ihrer Unwissenheit und persuasion von der so grossen Gewalt des Teuffels über cörperliche Dinge erhält/ und/ damit sie ja nicht etwan umfalle [...] dieselbe zu behaupten und defendiren mündlich und schrifftlich bemühet ist.

Da Schulze nicht nur der Schüler, sondern auch das Sprachrohr des allseits geschätzten Professors Hoffmann war, der den Rückhalt der Fakultäten in Halle genoss, schien es, als sei der Teufel an der dortigen Hochschule bereits

16 Johann Heinrich SCHULZE, Erdmann Friedrich ANDREAE Medicinae Doct. und Pract. in Jena, Sogenannter Gründlicher Gegensatz auf das ohnlängst in Halle ausgegebene Gründliche Bedencken und Physicalische Anmerckungen Von dem tödlichen Dampffe der Holtz-Kohlen/ etc. Mit einer Vorrede und kurtzen Anmerckungen/ Welche nicht nur den Unfug und Ungrund des Gegensatzes entdecken/ sondern zur Erläuterung der Haupt-Sache viel beytragen werden, o.O. o.J. [Halle, Renger, 1716]. 
vollständig entmachtet. Die Jenaer Medizinische Fakultät sah sich nun wiederum veranlasst, zugunsten ihres angegriffenen Mitgliedes Doktor Andreae zu intervenieren. Zur Besänftigung der Gegenseite in Halle wurde aber sogleich eingangs der anonymen Beantwortungsschrift festgestellt, dass in Jena niemand den berühmten Professor Hoffmann, der sich bekanntlich der Gunst des preußischen Königs erfreute, als Urheber der beanstandeten Abhandlung über den Kohlen-Dampf vermutet hatte. Auch wurde hinzugefügt, dass man an einer Weiterführung der Kontroverse unter keinerlei Umständen interessiert sei. ${ }^{17}$ In der Frage nach der Macht des Teufels gingen die Jenenser nun vorsichtig einen Schritt hinter Andreaes Thesen zurück. Es gab in der Tat keine Beweise dafür! Doch sei Andreae durchaus berechtigt gewesen, diese Behauptung aufzustellen, für die sich zwar keine Beweise, aber mannigfache Exempel beibringen ließen. In der Tat fänden sich in der Geschichte und in der Gegenwart Beispiele, "welche solche Umstände bey sich führen/ wodurch man allerdings behaupten kan/ daß der Teufel die Macht habe/ nicht allein indirecte den Todt derer Menschen zu befördern/ sondern auch dieselben directe zu tödten.” Anders als in Halle war man in Jena auch weiterhin nicht bereit, sich in die Entmachtung des Teufels ohne Widerspruch zu fügen.

\section{Der Versuch eines Schlussworts: Das Gutachten der drei Leipziger Fakultäten}

Angesichts des außergewöhnlichen Falles und des Streites gelehrter Autoren ersuchte die Eisenacher Regierung um eine entscheidende Stellungnahme der drei Leipziger Fakultäten, da die ausufernde Debatte zu einer Belastung für das Erscheinungsbild der Universität Jena zu werden drohte. Die Gutachten der Theologen, Juristen und Mediziner aus Leipzig wurden sogleich nach ihrer Verabschiedung gedruckt, um damit der Debatte den Wind aus den Segeln zu nehmen. ${ }^{18}$ Die Professoren der kursächsischen Hochschule waren sich bewusst, dass sie mit ihrer Einlassung unter starker Beobachtung durch die Öffentlichkeit standen:

17 C.A.T. Med. Cult. Unpartheyische Prüfung der Vorrede und kurtzen Anmerckungen Herrn Johann Heinrich Schultzens/Medic. Candidati, Womit Derselbe Den Herrn Autorem des ohnlängst in Halle ausgegebenen und von Herrn D. Andreae wiederlegten Bedenckens von dem tödtlichen Dampff der Holtz-Kohlen etc./ zu vertheidigen gesuchet, Jena, Werther, 1716.

18 Der drey Hohen Fakultäten zu Leipzig Bedencken und respective Urthel/ Welche Uber den zu Jena in der Heil. Christ-Nacht Anno 1715. passirten Traurigen Casum mit denen so genannten Teuffels-Bannern/ Auf Begehren derer Hoch-Fürstl. Weymarischen [sic!] Hrn. Commissarien/ den 6. Mart. 1716 ausgefertiget worden, o.O. o.J. [1716]; unvollständig hingegen die vorhergehende Veröffentlichung: Der theologischen, juristischen und medicinischen Facultäten in Leipzig Responsum über die Jenische Conjurations-Sache, o.O. 1716. 
Die genugsam bekannte Adjurations-Affaire zu Jena hat nicht allein in dem gemeinen Leben viel Gelegenheit zu reden/ sondern auch unter den Gelehrten viel Ursache zum Disputiren gegeben.

Es musste folglich mit Umsicht ein Urteil gefällt werden, das Gemeinen und Gelehrten einleuchtete. Um den Leipzigern die Entscheidungsfindung zu erleichtern, haben ihnen die aus Eisenach verordneten Kommissare die drei Bände umfassenden Untersuchungsakten aus dem Jenaer Stadtgericht zugeleitet, nebst einer versiegelten Schachtel mit Objekten aus dem Besitz des Studenten Weber, die dieser bei der versuchten Geisterbeschwörung benutzt hatte. Dazu gehörten vier magische Bücher, nämlich nicht näher bezeichnete Ausgaben der "Clavicula Salomonis", der "Philosophia occulta" Agrippas von Nettesheim, von "Dr. Fausts Höllen-Zwang" sowie eine nicht näher bezeichnete Edition der Schriften des Paracelsus.

Der Theologischen Fakultät ging es in erster Linie um die Frage der Sünden-Schuld Webers, der die "Geister-Citation" zugab und als einziger der an ihr Beteiligten überlebt hatte. Eine Schuld Webers erschien den Theologen in zweierlei Hinsicht gegeben: Bruch des Tauf-Bundes (Teufels-Pakt) und Blasphemie (Missbrauch von Gebetsformeln). Daher sollte er über seine Sünden belehrt werden. Eine andere Frage war diejenige einer Beteiligung des Satans an den Jenenser Ereignissen. Diese stand nicht fest, war doch bei den Opfern eine Erstickung "naturali modo durch Exhalation der Kohlen" erfolgt. Aber sie war auch nicht auszuschließen, da der Satan nach göttlicher Anordnung sein Werk auch unter Zuhilfenahme natürlicher Mittel verrichten konnte. Gegen cartesianische Auffassungen hielten die lutherischen Theologen daran fest, dass Geister in der Lage waren, auf die Materie einzuwirken. Sie schrieben dem Ereignis im Jenenser Weinberg eine moralische Aussagekraft zu. Es konnte sich dabei um ein verhängtes Schrecknis handeln, "um die sichern Welt-Leute/ die entweder sich nicht für dem Teuffel fürchten/ oder keine Teuffel glauben/ erschrocken zu machen über ihre Thorheit/ und der Heil. Schrift zu glauben/ als welche allen TeufelsDienern und Beschwerern [Beschwörern] den Tod gedrohet hat".

Für die Juristenfakultät war es hingegen überflüssig, nach übernatürlichen Ursachen für die Todesfälle in dem Weinbergshäuschen zu suchen, lagen doch hinlängliche natürliche Ursachen vor, die das Ereignis erklärten, da die Opfer allesamt durch den Holzkohlendampf erstickt waren. Als heidnische Praxis war die Geisterbeschwörung jedoch gegen die Ordnung des christlichen Staates gerichtet und daher strafwürdig. Folglich hatte die Eisenacher Regierung richtig gehandelt, wenn sie die Beisetzung der Leichen Geßners und Zenners unter dem Galgen anordnete. Die angemessene Strafe für den überlebenden, völlig mittellosen Studenten Weber bestand in der Verjagung von der Universität und seiner Ausschließung vom akademischen Studium auf Lebenszeit. Der Jenaer Bürger Heuchler, der sein Häuschen für den Akt der Geisterbeschwörung zur Verfügung gestellt und sich als Mitwisser schuldig gemacht hatte, sollte für zehn Jahre aus dem Land verwiesen und zur Übernahme der Prozesskosten verurteilt werden. Eine von 
den Kommissaren ebenfalls verhörte Bekannte Heuchlers mit Namen Margarethe Nitzschke sollte hingegen freigesprochen werden, da bei ihr eine Mitwisserschaft nach Aktenlage nicht nachgewiesen werden konnte.

Ähnlich wie die Juristen lehnten auch die Leipziger Mediziner ein Eingehen auf die Teufels-Hypothese ab. Es gebe genügend natürliche Ursachen zur Erklärung des Sachverhalts, so dass auf übernatürliche und vernunftwidrige Gründe ("causae abstrusiores") nicht näher einzugehen war. Die Ausdünstungen der Kohlen waren demnach allein für die drei Todesfälle verantwortlich zu machen. Allerdings sind die Opfer, entgegen verbreiteter Annahme, dabei nicht erstickt, sondern durch das in Lungen und Gehirn eindringende Gas ("Gas sulphuris") getötet worden. Einzelne Striemen an der Leiche Zenners, die bei der medizinischen Untersuchung festgestellt worden waren, können nicht als Beweis für eine äußere Gewaltanwendung mit möglicher Todesfolge angesehen werden. Wenn der dritte der aus Jena abgeordneten Wächter mit Namen Beyer ebenfalls verschieden ist, so mag dies auch eine Folge starker Gemütsbewegung aus Furcht vor dem Teufel gewesen sein. Außerdem verweisen die Leipziger Mediziner darauf, dass die Wächter nach eigener Aussage Branntwein getrunken hatten, so dass ihre vermeintlichen Beobachtungen in der Winternacht keinerlei Beachtung verdienten. Die Professoren in Leipzig waren offenkundig der Ansicht, dass der Teufel seine Macht über nüchterne Zeitgenossen bereits völlig eingebüsst haben musste.

\section{Im Rahmen der Naturgesetze: Der Machtverlust des Teufels}

Die Entmachtung des Teufels war am Anfang des 18. Jahrhunderts in ganz Europa ein Gegenstand leidenschaftlicher Debatten, wobei sich die Mediziner auch bereits in Frankreich als Meinungsführer vor den Theologen und Juristen hervorgetan hatten. ${ }^{19}$ Dabei ging die Mehrzahl der Einlassungen von medizinischer Seite eher von natürlichen Ursachen als von dämonischen Phänomenen aus, doch hielt sich auch bei den Vertretern der Heilkunde lange Zeit die Auffassung, dass der böse Geist weiterhin in das menschliche Tun und Treiben hineinwirkte. Insofern war seine Macht noch keineswegs verschwunden. Die Suche nach natürlichen Ursachen für rätselhafte Vorfälle schloss die Vermutung des Übernatürlichen nicht a priori aus. Daher waren die Diskussionen in den ersten beiden Jahrzehnten des 18. Jahrhunderts in einem Übergangsfeld situiert. Auch für Frankreich konnte in dieser Zeit eine lebhafte Publikationstätigkeit zum Gegenstand nachgewiesen werden, bei der

19 Vgl. dazu besonders: Nadia MinERVA, Il Diavolo. Eclissi e Metamorfosi nel secolo dei Lumi. Da Asmodeo a Belzebù, Ravenna, Longo, 1990, S. 50-57. Daneben auch: Jean-Marie GOULEMONT, "Démons, merveilles et philosophie à l'âge classique", in : Annales ESC 3 (1980), S. 1223-1250. 
es stets um die brennende Frage nach der Macht des Leibhaftigen ging. ${ }^{20}$ Konnte der Teufel Menschen töten oder zumindest Körper verletzen? Zwar war Satan um 1710 in Europa auf dem besten Weg, um zum "Dämon des Wahnsinns" zu werden, ${ }^{21}$ zum Beherrscher der Einbildungskraft, doch hatte er noch nicht alle Macht in den Ordnungssystemen der Vernunft verloren. In den Diskursen konnte ihm ein Platz unter den Dingen dieser Welt zugewiesen werden. Dabei ist auffällig, in welchem Maße sich die Vertreter medizinischer Gelehrsamkeit in der Diskussion hervortaten, indem sie versuchten, die Frage nach der Macht des Leibhaftigen überzeugender zu klären, als dies die Theologen oder ebenso die Juristen bis dahin vermocht hatten.

Als Beispiel für diese Klärungsversuche aus dem Bereich medizinischer Gelehrsamkeit lässt sich wiederum der Hallenser Hochschullehrer Friedrich Hoffmann heranziehen, in dessen Denken sich die genannten Spannungszustände abgebildet finden. Mochte Hoffmann auch mit seiner Einlassung zu der "Christnachtstragödie" von 1715 der Debatte eine Wendung zum Diskurs über die "natürlichen Ursachen" gegeben haben, so ginge seine Vereinnahmung für "cartesianische" Ansichten doch vollkommen an seiner Ideenwelt vorbei. Er ist somit eine hervorragende Zeugenfigur für die Zeit der Übergänge des Teufelsdiskurses im Jahrzehnt des Jenaer Ereignisses. Bereits im Jahr 1712 hatte Hoffmann eine Untersuchung zu der Frage veröffentlicht, ob der Teufel Macht über den menschlichen Körper ausübe und damit der von dem späteren Ereignis ausgelösten Debatte vorgegriffen. ${ }^{22}$ Für ihn gab es demnach immer noch einen gültigen Konsens der Theologen, Physiker und Mediziner über die Natur des Teufels, demzufolge dieser ein böser Geist sei, der Gewalt über andere Kreaturen, zumal über den Menschen, ausübe. ${ }^{23}$ Diese Gewalt wirkt jedoch vor allem auf die menschliche Einbildungskraft ein. ${ }^{24}$ Der Einzelne ist frei, sich dieser Einwirkung zu widersetzen, doch kann er ihr ebenso gut aus Schwäche unterliegen. Auf diesem Umweg über die menschliche Vorstellungskraft kann

20 Robert Muchembled, Une Histoire du diable. XII $-X X^{e}$ siècle, Paris, Seuil, 2000, S. 223-232. Vgl. die angegebenen Buchtitel bei: Robert YVE-PLESSIS, Essai d'une bibliographie française méthodique et raisonnée de la sorcellerie et de la possession démoniaque, Paris, Bibliothèque Chacornac, 1900 (ND Genf, Slatkine, 1970).

21 MinerVA, Il Diavolo, S. 52.

22 Friedrich Hoffmann, De Diaboli Potentia in Corpora, dissertatio physico-medica curiosa, Halle, Gruner, 1712 (unpaginiert). Die wesentlichen Gedanken Hoffmanns zu dem Gegenstand finden sich bereits in einer Inauguraldisputation von 1703: Friedrich HOFFMANN/ Gottfried BüchIng, Disputatio inauguralis medico-philosophica, De Potentia diaboli in Corpora, Halle, Gruner, 1703. Der Respondent Gottfried Büching hat selbst eine deutsche Übersetzung des Textes herausgebracht: G.B.M.D. Philosophische Untersuchung von Gewalt und Wirckung des Teuffels in natürlichen Cörpern, Frankfurt/ Leipzig 1704.

23 Friedrich Hoffmann, De Diaboli Potentia (1712), § 1: "Est autem diabolus, communi omnium cum theologorum tum physicorum \& medicorum consensu, spiritus creatus finitus, nocentissimus, certa potentia in creaturas, maxime in hominem, praeditus".

24 Ibid.: "Quapropter in eam animae partem, quae phantasia dicitur, mira ejus est energia". 
Satan auch die Körper zu einem bestimmten Handeln bewegen, doch ist ihm die direkte Einwirkung auf die Materie versagt. Wunder kann er nämlich nicht verrichten und die Auslösung einer natürlichen Wirkung durch eine übernatürliche Ursache wäre ein solches. Insofern sind der Macht des Leibhaftigen durch die von Gott verordneten Gesetze der Natur enge Grenzen gesetzt, die er nicht aufheben kann. Der Böse hat Macht allenfalls im Rahmen der Naturgesetze, sofern es Menschen gibt, die ihm als willige Gehilfen dienen. Daher ist alles falsch, was man beispielsweise über dessen Vermögen erzählt, menschliche Körper durch die Luft zu transportieren. Dieses wäre ein Wunder und steht ihm somit nicht zu. Ebenso wenig vermag er es, die materielle Qualität der Dinge zu verändern, zum Beispiel aus unedlen Metallen Gold zu machen, wie dies immer wieder behauptet wird. Er kann sich auch nicht direkt der Körper bemächtigen, allenfalls vermag er es, über die Geister Gewalt zu gewinnen. So ist für den Hallenser Mediziner auch Satans Reich nicht von dieser Welt. Als Geist herrscht er vielmehr in den Lüften. Hier kann er Stürme und Unwetter auslösen. ${ }^{25}$ An der Macht des Teufels, die Ernten durch schlechtes Wetter und andere Unglücksfälle zu schädigen, will Hoffmann ausdrücklich festgehalten wissen! So wird dem Teufel gleichsam ein eigener Bereich zugewiesen, in dem er seine Macht wie ein konstitutioneller Herrscher im Rahmen der Gesetze ausüben kann, nämlich der Makrokosmos der Luft und der Mikrokosmos der menschlichen Einbildungskraft. ${ }^{26}$ Ganz wie es Malebranche oder Fontenelle vor ihm gemutmaßt hatten, so nimmt auch Friedrich Hoffmann an, dass die Berichte der Hexen und Geisterbeschwörer von Begegnungen mit dem Dämon reine Phantasiegebilde seien. ${ }^{27}$ Luft und Äther, Schein und Wahn sind die Zonen, in denen teuflische Macht sich zäh behauptet.

Freilich bleibt es den Menschen freigestellt, inwieweit sie sich dieser Macht unterwerfen wollen. Dabei spielen auch psychische Prädispositionen und kulturelle Besonderheiten eine Rolle. Nach Ansicht des Mediziners, der vor seiner Berufung nach Halle 1693 unter anderem als Landphysikus im Fürstentum Minden praktiziert hatte, waren die Biertrinker Westfalens viel anfälliger für solche Teufelsvisionen als die heiteren und rationalen Weintrinker in Frankreich oder Italien $!^{28}$ Bei jenen, die ihm erliegen, kann der Leibhaftige auch Krankheiten auslösen und somit eine direkte Macht über den menschlichen Körper gewinnen, doch handelt es sich dabei in erster Linie um Krankheiten des Geistes wie Tobsucht, Melancholie oder Epilepsie. Dabei ist aber daran festzuhalten, dass solche Krankheiten ebenso rein natürlichen Ursachen entspringen können. Es entsprach der historischen Entwicklung, wie Hoffmann sie verstand, wenn die natürlichen Ursachen immer mehr die

25 Ibid., § XIII: "Porro cum imperium \& dominatum habeat in aerem, non negari poterit".

26 Ibid., $\S \mathrm{XV}$.

27 Ibid., § XVI: "Ita pleraeque operationes diaboli in sagis sunt merae illusiones phantasticae".

28 Ibid., § XVII. 
Überhand gewannen. Er sah die Logik der Geschichte am Werk, die der Macht des Dämons am Anfang des 18. Jahrhunderts entgegen arbeitete. Dies verstand er als eine langfristige Folge der die Menschen befreienden Erlösungstat Christi, so dass die Teufelserscheinungen insgesamt in der Gegenwart immer seltener wurden. Satans Macht wird somit nach und nach auch in den ihm noch verbleibenden Machtbereichen gebrochen! ${ }^{29}$ Eines Tages, so die zuversichtliche Erwartung Hoffmanns, wird sie ganz verschwunden sein, da sich das Licht der Wahrheit immer mehr in den Gemütern der Menschen ausbreitete, Wissenschaft und Künste immer mehr aufblühten. ${ }^{30}$ Sicherlich wird es den Teufel als von Gott geschaffene Kreatur und per se unsterblichen Geist immer geben, bis an der Welt Ende, doch wird er irgendwo in dem ihm verbliebenen Reichsteil der Lüfte eine trübsinnige Geisterexistenz führen. Wenn die Menschen nicht mehr an ihn glauben, dann wird seine Macht auf der Welt dahin sein. Dies konnte für einen großen Mediziner am Anfang des 18. Jahrhunderts auch eine mögliche Sinngebung von "Aufklärung" sein.

29 Ibid., § XXV: "Daemones hodie non tanto imperio tantaque potestate uti in rerum naturalium ordinem, ipsumque genus humanum, quanta olim polluit. Quod vel inde apparet, quod nostris temporibus spectra, obsessiones, incantationes, aliaeque hujus generis praestigiae rariores sunt quam olim".

30 Ibid., § XXV: "Neque dubitamus, fore, ut inposterum ejus potentia ludibriaque magis magisque evanescant. Clarior enim lux veritatis ubique in animis hominum coepit exsplendescere, florent artes \& scientiae, rationis cultura ubique". 